\title{
Avaliação da actividade física em contextos epidemiológicos. Uma revisão da validade e fiabilidade do acelerómetro Tritrac-R3D, do pedómetro Yamax Digi-Walker e do questionário de Baecke.
}

\author{
M. M. Oliveira
}

J. A. Maia

Faculdade de Ciências do Desporto e de Educação Física

Universidade do Porto

Portugal

https://doi.org/10.5628/rpcd.01.03.73

\section{RESUMO}

A actividade física é um fenómeno complexo cuja avaliação se reveste de dificuldades ao nível da validade e precisão de medição. Na literatura podemos encontrar uma diversidade de metodologias possíveis, que se prendem com o vasto conjunto de dimensões que este fenómeno apresenta. O propósito do presente trabalho é rever e sintetizar o estado actual do conhecimento acerca da fiabilidade e validade do acelerómetro Tritrac-R3D, do pedómetro Yamax Digi-Walker e do questionário de Baecke para a avaliação da actividade física em contextos epidemiológicos. Os resultados dos estudos revistos evidenciam que: (1) o acelerómetro é um instrumento válido para a avaliação da actividade física habitual em termos de intensidade, frequência e duração, em populações onde a marcha e a corrida ou outros movimentos semelhantes que impliquem o deslocamento do centro de massa sejam os mais representativos do padrão de actividade física habitual; (2) o pedómetro fornece indicações válidas e objectivas da actividade física habitual, se bem que só meça a que se relaciona com a deslocação a pé; (3) os questionários são, sem sombra de dúvida, os instrumentos mais práticos e comuns em estudos de carácter epidemiológico em virtude dos seus baixos custos e da sua enorme praticabilidade, ainda que o carácter objectivo da sua informação seja questionável, não obstante a sua validade e fiabilidade.

Palavras-chave: actividade física, validade, fiabilidade, acelerómetro, pedómetro, questionário.

\begin{abstract}
Assessment of Physical Activity in Epidemiological Context. A Review of the Validity and Reliability of the Tritrac-R3D Accelerometer, Yamax Digi-Walker Pedometer and Baecke Questionnaire.

Physical activity is a complex phenomenon whose assessment is filled with difficulties concerning its validity and reliability. Several methodologies are available to measure different components of physical activity. The main purpose of this report is to update the state of the art about validity and reliability of an accelerometer (TRITRAC), a pedometer (Yamax) and a questionnaire (Baecke) used in epidemiological contexts. Main results from reviewed studies point to the following: (1) the accelerometer is a valid and reliable instrument to capture different facets of daily physical activity in terms of intensity, frequency and duration be it in walking, running or any other movement that implies displacement of the centre of gravity of the subjects; (2) the pedometer reproduce valid and reliable information regarding walking, only; (3) the questionnaire is undoubtedly the most practical and used instrument in epidemiological research, whose main virtues are the low cost and high practicability, but whose objectivity may be somewhat low, regardless of its validity and reliability.
\end{abstract}

Keywords: physical activity validity, reliability, accelerometers, pedometers, questionnaires. 


\section{ACTIVIDADE FÍSICA \\ Conceito}

Um dos aspectos importantes em qualquer pesquisa no lato domínio da avaliação da actividade física é, sem sombra de dúvida, o da exigência de uma definição clara e inequívoca do seu conteúdo.

A actividade física é um fenómeno/comportamento extremamente complexo $(13,35,42)$, sendo actualmente considerado como um conjunto de comportamentos que inclui todo o movimento corporal (42) a que se atribui um significado díspar em função do contexto em que é realizado.

O conceito de actividade física tem sido objecto de alterações ao longo do tempo e de todas as definições que se podem encontrar na literatura, seleccionamos a de Caspersen e col. (13) por ser a que mais consensos reúne $(9,15,27,28,37)$. Actividade física é entendida como qualquer movimento corporal produzido pelos músculos esqueléticos que resulte em dispêndio energético. Assim, toda e qualquer actividade física protagonizada pelo sujeito no seu dia-a-dia cabe nesta abrangência, já que contribui para o dispêndio energético total, isto é, a actividade física utilizada nas deslocações, nas tarefas da vida diária, nas actividades em tempo de lazer, nas actividades desportivas mais ou menos organizadas, no trabalho e, no caso das crianças, na escola e nas suas actividades lúdicas.

Dado que a existência humana obedece à lei da conservação da energia, a medição e quantificação da actividade física é frequentemente expressa em termos de dispêndio energético (35), se bem que sejam utilizadas, também, outras possibilidades. A quantidade de energia necessária para a realização de uma actividade pode ser medida em Kilocalorias (Kcal) ou Kilojoules (Kj). Em alternativa, a actividade física pode ser expressa em watts, como a quantidade de trabalho produzido, em minutos e horas que indicam períodos de tempo de actividade, como contagens de movimento (counts) que representam unidades de movimento, ou até como um valor numérico obtido a partir das respostas dadas a um questionário (35).

O dispêndio energético total associado à actividade física é determinado pela quantidade de massa mus- cular envolvida, pela intensidade, duração e frequência das contracções musculares. Embora todos nós sejamos mais ou menos activos, o valor total da actividade física depende fundamentalmente daquilo que cada um realiza durante um determinado período de tempo; este valor evidencia uma forte variação interindividual, que se comporta de acordo com a distribuição normal (13). Importa esclarecer que a expressão dispêndio energético não deve ser usada como sinónimo de actividade física. Em primeiro lugar porque, apesar do dispêndio energético ser uma consequência da actividade física, quando falamos do dispêndio energético total incluímos outras componentes como sejam a taxa de metabolismo basal e o efeito térmico induzido pela assimilação e digestão dos alimentos. Em segundo lugar, porque a quantidade de energia despendida por um indivíduo é entendida sempre como um valor relativo, equacionado em função da sua massa corporal. Um indivíduo dimensionalmente pequeno que seja extremamente activo pode gastar, diariamente, a mesma quantidade de Kilocalorias que um indivíduo dimensionalmente maior e que seja sedentário. Daqui que não se possa afirmar que sejam semelhantes em termos de actividade física (27). Por isso, sempre que a actividade física for expressa em termos de dispêndio energético, Kilocalorias ou Joules, a massa corporal do indivíduo tem que ser tomada em consideração (35). A expressão MET (abreviatura de equivalente metabólico) é utilizada para significar o dispêndio energético em função do peso do sujeito. Por equivalente metabólico entende-se o valor correspondente à energia despendida em repouso, representando este valor uma referência para a classificação da intensidade das actividades (35). Assim sendo, os valores de dispêndio energético são expressos em múltiplos de METs, correspondendo um MET a um gasto equivalente ao valor de energia metabólica despendida pelo sujeito em repouso, por quilo de peso, isto é, $3.5 \mathrm{ml} \mathrm{O}_{2} \cdot \mathrm{Kg}^{-1} \cdot \min ^{-1}(21,33)$.

A actividade física é frequentemente entendida como um constructo multidimensional onde se incluem variáveis como o tipo, a frequência, a intensidade, a duração e, embora menos referida, a circunstância e 
o propósito da actividade $(9,35)$. Segundo estes últimos autores, tanto o envolvimento físico (por ex.: as circunstâncias em que se realiza a actividade, isto é, altitude, temperatura ambiente, etc.) como o conteúdo emocional ou psicológico, podem alterar os efeitos fisiológicos de uma actividade. A frequência, a intensidade e a duração podem ser combinadas para providenciarem informações acerca da quantidade de energia gasta na actividade física.

Importa salientar que o dispêndio energético diário total é composto por 3 componentes $(33,35)$ :

- a taxa de metabolismo basal, isto é, a energia necessária para o corpo manter a sua temperatura, bem como as contracções musculares involuntárias;

- o efeito térmico da alimentação, isto é, a energia necessária à digestão e assimilação dos alimentos;

- a quantidade de energia despendida na actividade física, isto é, todo o movimento corporal realizado voluntariamente e durante todo o dia.

As duas primeiras componentes apresentam uma variabilidade inter- e intra-individual pequena (o seu contributo no dispêndio energético diário total é de cerca 60 a $75 \%$ ) comparativamente com a maior fonte de variação que é, sem sombra de dúvida, a que advém da enorme variabilidade dos valores da actividade física dos sujeitos $(8,35)$.

\section{MÉTODOS DE AVALIAÇÃO}

A avaliação de um comportamento tão complexo como a actividade física reveste-se de complexidades e dificuldades ao nível da precisão da sua medição (22). Na literatura podemos encontrar uma grande diversidade de metodologias para avaliar a actividade física. Essa diversidade é, pelo menos em parte, justificada pelo vasto conjunto de dimensões que o fenómeno apresenta e pelas complexas relações que cada uma delas estabelece com diferentes aspectos da saúde $(28,35)$. De acordo com a dimensão da actividade física que se pretende estudar, deverá emergir uma diferente operacionalização do conceito assim como o instrumento de medição mais apropriado. Cada uma das abordagens na avaliação é susceptível de medir apenas uma parte do padrão do comportamento global (28). Por exemplo, enquanto uns instrumentos medem o dispêndio energético, outros medem a frequência, a duração, a intensidade ou o tipo de actividade.

A escolha dos instrumentos de avaliação deverá ser governada pelos objectivos da pesquisa $(27,35)$, estar dependente das idades dos participantes (27) e de questões de ordem prática que se prendem com custos, tempo para a realização do estudo, aceitabilidade do instrumento por parte da amostra e exequibilidade (28). Para além destes factores, há ainda a considerar a circunstância da opção dever também ter por base a validade, fiabilidade e praticabilidade do instrumento (22). Para ser válido, o instrumento deve medir aquilo que se propõe medir; para ser fiável deve ser consistente perante as mesmas circunstâncias; se for fidedigno e válido é também preciso (28); para ser prático deve ter "custos" aceitáveis tanto para o investigador como para o participante. Outras considerações deverão incluir a intrusão provocada pelo instrumento, a sua influência nos níveis de actividade física dos participantes, as possíveis falhas e a "contaminação" ou alteração dos dados $(2,35)$.

Torna-se, portanto, evidente, quão difícil é medir com precisão um fenómeno que é multidimensional por natureza, e que é passível de ser avaliado de diferentes formas. Tornar-se-á claro também que qualquer técnica ou instrumento de avaliação mede somente uma parte do "todo" do fenómeno actividade física habitual $(5,35)$. Daqui que uma avaliação mais completa reclame uma recolha de informação associada às diferentes dimensões (5), e que seja multimodal na sua instrumentação.

Os métodos existentes podem ser divididos em duas grandes categorias: os métodos laboratoriais e os métodos de terreno. No quadro $n .{ }^{\circ} 1$ é apresentada uma listagem desses métodos segundo as indicações de Laporte e col. (28), Caspersen (12) e Montoye e col. (35). Descrições mais detalhadas acerca dos mesmos podem ser encontradas na literatura mencionada. 
Quadro 1: Sumário dos métodos de avaliação da actividade física - adaptado de Laporte e col. (28), Caspersen (12) e Montoye e col. (35).

\begin{tabular}{ll} 
Métodos Laboratoriais & Métodos de terreno \\
\hline 1. Fisiológicos & 1. Diário \\
- Calorimetria directa & 2. Classificação profissional \\
- Calorimetria indirecta & 3. Questionários e entrevistas \\
& 4. Marcadores fisiológicos \\
2. Biomecânicos & - "Double Labeled Water" \\
- Plataforma de força & - Aptidão cárdio-respiratória \\
& 5. Observações comportamentais \\
& 6. Monitorização mecânica \\
& e electrónica \\
& - Sensores do movimento \\
& Pedómetro \\
& Acelerómetro \\
& - Monitores de frequência cardíaca \\
& 7. Aporte Nutricional
\end{tabular}

Na categoria relativa aos métodos laboratoriais, encontramos os procedimentos mais objectivos e precisos, mas que exigem equipamentos sofisticados e dispendiosos para além de processos de análise de dados bastante complexos. A maior parte destes métodos não pode ser aplicada em estudos epidemiológicos, mas servem de critério de validação para os métodos de terreno. No segundo grupo, encontram-se os métodos de terreno, menos complexos, aplicáveis em contextos diferenciados e em amostras de grande amplitude, porém, menos precisos (35). Esta é uma matéria de alguma controvérsia dada a inexistência de um método universalmente aceite que meça todas as componentes da actividade física em condições de free living.

$\mathrm{O}$ conhecimento actual na área da actividade física, principalmente o de carácter epidemiológico, baseia-se em estudos que recorreram aos questionários de actividade física devido aos seus baixos custos e facilidades de implementação. Contudo, é evidente que possuem limitações inerentes, já que são por natureza subjectivos (5). Torna-se pois necessário explorar métodos alternativos que não estejam dependentes da aptidão do sujeito em recordar o seu padrão de actividade, especialmente em idades muito jovens. Os sensores do movimento têm a potencialidade de eliminar este tipo de problemas (21).
Um grande número de aparelhos mecânicos e electrónicos têm vindo a ser desenvolvidos com o intuito de trazer objectividade e precisão às medições. Dentre os instrumentos que monitorizam o movimento, encontram-se os acelerómetros e os pedómetros, métodos alternativos aos questionários, que medem a aceleração corporal e estimam o dispêndio energético associado. Os pedómetros apenas quantificam o movimento e os acelerómetros medem simultaneamente a quantidade, intensidade e direcção do movimento (28). Apresentamos de seguida informações mais pormenorizadas acerca destas ferramentas.

\section{Acelerómetros}

Os acelerómetros são sensores do movimento, sensíveis a variações na aceleração do corpo num ou nos três eixos e, por isso, capazes de providenciar uma medição directa e objectiva da frequência, intensidade e duração dos movimentos referentes à actividade física realizada (10). Quando um sujeito se move o corpo sofre uma aceleração, teoricamente proporcional à força exercida pelos músculos responsáveis por essa aceleração e, por isso, proporcional à energia despendida (35). A avaliação da actividade física através da medição do movimento do sujeito é cada vez mais apelativa porque, à partida, toda a actividade física requer movimento; os mais activos movem-se mais do que os menos activos e, por outro lado, a quantidade de movimento é, provavelmente, mais precisa na avaliação da actividade física do que a estimação do dispêndio energético (28). Os sensores do movimento são actualmente utilizados para quantificar uma generalidade de movimentos realizados quotidianamente e para efectuar estimativas do dispêndio energético correspondente.

Os acelerómetros estão cada vez mais disponíveis no mercado em menores dimensões; por este motivo são mais práticos e também tecnologicamente mais sofisticados, providenciando informações mais precisas. $\mathrm{O}$ acelerómetro uniaxial mede a aceleração corporal apenas no eixo vertical, enquanto que o triaxial detecta a aceleração em 3 eixos (X, Y e Z). Tendo em conta que a movimentação do corpo é pluridireccional, vários autores indicam como método mais apropriado para a avaliação da actividade física e do dispêndio energético a medição nos três eixos, comparativamente com a medição do movimento corpo- 
ral num só eixo $(20,29,35)$. Outros autores referem que actividades esporádicas como as realizadas pelas crianças podem não ser adequadamente medidas através de um aparelho unidimensional (ver por exemplo 46).

Os avanços na tecnologia conduziram ao desenvolvimento de sensores do movimento mais sofisticados tal como o TRITRAC - R3D (medidas: $11 \times 6.9$ x $3.3 \mathrm{~cm}$; peso: $170 \mathrm{~g}$ ). Este acelerómetro avalia a aceleração corporal em três eixos (antero - posterior, médio - lateral e vertical) e providencia um valor composto resultante, designado de vector magnitude ou vector resultante. Efectua os registos de actividade em intervalos de tempo que variam de 1 a 15 minutos, tem uma capacidade de armazenamento da informação de 31 dias e é alimentado por uma bateria de 9 volts (para pormenores ver 35). Este instrumento tem sido proposto como um método adequado de avaliação da actividade em crianças e outros grupos populacionais $(10,16,17,43)$.

As vantagens deste aparelho incluem: (i) a possibilidade de ser aplicado a qualquer escalão etário; (ii) o facto de ser compatível com as actividades quotidianas, permitindo assim avaliar os sujeitos em condições reais de vida; (iii) a grande capacidade de armazenamento de dados; (iv) a não existência de comandos que possam ser manipulados externamente e (v) a capacidade de avaliar a actividade durante períodos de tempo específicos, possibilitando a análise da frequência, duração e intensidade. Contudo, têm também algumas limitações. O dispêndio energético não aumenta à medida que aumenta a carga de trabalho, nomeadamente com o levantamento de pesos ou com o trabalho estático (26); tendem a sobrestimar o dispêndio energético na marcha e a subestimar esse gasto associado a muitas outras actividades; são imprecisos na estimação do dispêndio energético associado à posição de sentado e nos movimentos da parte superior do tronco e não podem ser usados em actividades aquáticas (5). Para além das limitações referidas, Croker e col. (18) enumeraram outras relativas à praticabilidade do aparelho. Este autor realizou recentemente um estudo com a intenção de avaliar precisamente questões relacionadas com este problema. Foi realizado em 79 crianças com uma média de 11 anos de idade que usaram o TRITRAC - R3D durante 7 dias consecutivos. Apesar dos telefonemas diários e das visitas às escolas, a maior parte dos participantes teve os seguintes problemas: (i) esquecimento na colocação; (ii) desconforto no seu uso e embaraço público; (iii) impossibilidade de uso durante a realização de actividades aquáticas e (iv) a não autorização para uso durante as actividades físicas organizadas. Os resultados colocam algumas reticências quanto à viabilidade do TRITRAC para a avaliação da actividade física durante períodos extensos de tempo, sobretudo neste grupo etário. Convém, contudo, realçar a circunstância de condicionalismos culturais da pesquisa anterior, bem como a ausência de forte generalização das conclusões face à dimensão reduzida da amostra. Outros estudos não têm encontrado tais limitações (ver por exemplo 17, 19, 46, 47).

\section{Estudos de validade e fiabilidade}

Diversos estudos examinaram a validade da acelerometria e especificamente do TRITRAC-R3D na avaliação da actividade física e do dispêndio energético sob diferentes perspectivas em amostras de crianças e jovens $(17,19,20,30,46,47)$, e em adultos (10, $16,26,36,43,45)$.

Por validade entenda-se o grau de precisão com que o instrumento mede ou avalia o que se propõe medir ou avaliar $(27,28,32)$. A determinação da validade realiza-se comparando os resultados com os valores de outro método-critério, de preferência um "gold standard". Relativamente às questões de validade, Kohl e col. (27) referem que estudos sobre instrumentos de avaliação desenhados para avaliar um constructo (actividade física), que são validados recorrendo ao uso de ferramentas que avaliam um outro constructo (por exemplo o dispêndio energético), estão com certeza limitados no total de concordância ou validade esperada por causa da combinação imprecisa do método de avaliação e do critério de validação.

Dois estudos recentes usaram a frequência cardíaca como critério de validação do TRITRAC - 3RD na avaliação da actividade física de crianças em circunstâncias reais de vida $(17,46)$.

Welk e Corbin (46) investigaram a validade concorrente do TRITRAC - 3RD em crianças dos 9 aos 11 anos de idade. As correlações encontradas foram moderadas $(\mathrm{r}=0.50)$ para os 3 dias de monitorização. Contudo, quando os autores fizeram a divisão do dia nas suas diferentes partes, observaram 
variações nas correlações entre as medidas, dependendo do tipo e duração da actividade das crianças. As correlações eram mais elevadas nas actividades espontâneas, onde as crianças tinham oportunidade de ser mais activas ( $r=0.83$ a $r=0.89)$ e mais baixas quando a actividade era menor (sala de aula, $r=$ 0.41) ou era estruturada (aula de educação física, $r$ $=0.69$ ). Os autores concluíram, face aos resultados, que o TRITRAC era um instrumento válido na avaliação da actividade física diária das crianças.

Coleman e col. (17) reportaram a validade do TRITRAC - 3RD na avaliação da actividade física nos tempos livres de crianças obesas (8 a 12 anos). Os dados do TRITRAC foram convertidos em METs e a correlação encontrada com a frequência cardíaca foi de 0.71 . Os resultados do estudo sugeriram que o vector magnitude do TRITRAC poderia providenciar uma estimativa mais compreensível da actividade física do que o vector do eixo vertical.

Welk e Col. (47) propuseram-se determinar qual dos "indicadores", frequência cardíaca ou TRITRAC, providenciava a medição mais válida em duas situações de avaliação, usando como medida critério a observação directa (Childrens Activity Record System CARS). Os resultados sugeriram que os dois instrumentos providenciavam uma informação similar, mostrando o TRITRAC ser um "instrumento-indicador" válido da actividade física em situações de maior e menor actividade (uma situação mais activa protagonizada pela aula de educação física e outra menos activa em sala de aula).

Eston e col. (20), num estudo realizado com o propósito de identificar os coeficientes de validação de vários instrumentos na avaliação da actividade física em 30 crianças galesas (idade: 8 a 10 anos), concluíram que a acelerometria triaxial providencia a melhor avaliação da actividade física, já que mostrou as correlações mais elevadas com o consumo de oxigénio, medida critério utilizada pelos investigadores $(\mathrm{r}$ $=0.90$ ). Louie e col. (30) chegaram às mesmas conclusões quando aplicaram o mesmo estudo numa amostra de 31 crianças chinesas com idades compreendidas entre 8 e 10 anos. Ambos os estudos encontraram correlações mais elevadas entre o vector magnitude do TRITRAC e a medida critério.

A "double labeled water", método considerado "gold standard" na determinação do dispêndio energético total em condições reais de vida, foi utilizada como medida-critério de validação num estudo recentemente realizado por Ekelund e col. (19) em crianças com uma média de 9.1 anos de idade. Os investigadores propuseram-se validar, ao longo de duas semanas, o acelerómetro uniaxial Computer Science and Aplication's (CSA) para a avaliação da actividade física. Como segundo objectivo, pretenderam desenvolver equações de predição do dispêndio energético total e do dispêndio energético na actividade, a partir de valores das contagens de actividade (counts) providenciados pelo acelerómetro e também de variáveis antropométricas. Os autores concluíram que as contagens de actividade do acelerómetro providenciavam dados válidos para se avaliar a quantidade total de actividade física em crianças de 9 anos de idade; que contribuíram significativamente para a explicação da variância no dispêndio energético total e que foram o melhor preditor do dispêndio energético na actividade física.

Em adultos e em condições laboratoriais foram realizados vários estudos de validação de que destacamos os mais actuais. Chen e Sun (16) realizaram uma pesquisa profunda para avaliar a validade da estimativa do dispêndio energético do TRITRAC-R3D, recorrendo a uma câmara de respiração (calorimetria indirecta) como critério de validade. Os 125 sujeitos testados, 53 homens e 72 mulheres dos 19 aos 62 anos, viveram durante dois dias nesta sala em condições "semelhantes" ao seu dia-a-dia. No primeiro dia viveram segundo o seu padrão de actividade física "normal" (dia normal) e no segundo executaram alguns exercícios, tais como corrida e "stepping" num período de tempo pré determinado (dia de exercício). A estimativa do dispêndio energético do TRITRAC para os dois dias avaliados esteve altamente correlacionada com o dispêndio energético total medido por calorimetria $(r=0.93)$, o mesmo se passando numa análise tendo por base o dispêndio energético minuto a minuto $(\mathrm{r}=0.86)$. $\mathrm{O}$ dispêndio energético formulado pelo TRITRAC na situação de repouso, foi semelhante aos valores medidos por calorimetria indirecta. Contudo, verificou-se que o TRITRAC subestimou o dispêndio energético total, tanto no dia considerado normal como no dia de exercício. Subestimou, também, o dispêndio energético na actividade física de uma forma geral, assim como nos casos específicos de actividades físicas sedentárias e de baixa intensidade. 
Sherman e col. (43) realizaram uma pesquisa em 10 homens e 6 mulheres com uma média de idades de $24 \pm 3$ anos, com o propósito de avaliar a capacidade do TRITRAC-R3D para a medição do dispêndio energético nas seguintes situações: repouso antes do exercício, marcha no tapete rolante e repouso logo após o exercício. Os autores encontraram uma relação significativa entre o dispêndio energético derivado da calorimetria indirecta e o dispêndio energético obtido no acelerómetro $(r=0.96)$. Daqui que tenham concluído que o acelerómetro usado mediu de forma precisa o dispêndio energético, excepto na situação de repouso logo após o exercício. Tal facto ficou, provavelmente, a dever-se às limitações do acelerómetro para estimar com precisão o dispêndio energético associado ao declínio no consumo de oxigénio após o esforço.

Welk e col. (45) investigaram a validade relativa (concorrente) e absoluta de 3 sensores do movimento contemporâneos: TRITRAC, CSA e Biotrainer. O estudo foi realizado perante circunstâncias laboratoriais e de terreno em 52 adultos de ambos os sexos com uma média de idades de 29 anos, servindo a calorimetria indirecta de medida-critério para testar a validade dos aparelhos. Os participantes realizaram duas rotinas que pretendiam simular um conjunto de actividades da vida diária. A correlação entre os aparelhos e o consumo de oxigénio foi superior na circunstância laboratorial comparativamente com a de terreno. No laboratório, o CSA produziu estimativas de dispêndio energético precisas, enquanto que o TRITRAC e o Biotrainer tenderam a sobrestimar o dispêndio energético (1 a 36\%). Contudo, verificou-se que o TRITRAC possuía um erro menor na estimação individual do dispêndio energético. No terreno, todos os aparelhos subestimaram o dispêndio energético (entre 42 e 67\%). Os autores concluíram que cada aparelho produziu informações semelhantes a avaliar pelas correlações encontradas entre si, tanto no laboratório como no terreno.

Dois estudos foram realizados com o propósito de examinar tanto a validade como a fiabilidade do TRITRAC-R3D em amostras constituídas por jovens adultos $(26,36)$. Quando falamos de fiabilidade estamos a referir-nos à consistência dos resultados de um instrumento (repetibilidade), ou seja, se apresenta os mesmos resultados perante as mesmas circunstâncias na medição de uma variável $(27,28)$.
Jakicic e col. (26) examinaram a validade e a fiabilidade do TRITRAC-R3D na estimação do dispêndio energético em várias formas de exercício em laboratório (marcha no tapete rolante, corrida no tapete rolante, subida e descida de degraus ("stepping"), pedalagem em bicicleta ergonómica e "slideboard"), usando como medida-critério de validação a calorimetria indirecta. A amostra foi constituída por 14 homens e 6 mulheres com idades compreendidas entre os 18 e os 35 anos. Os resultados mostraram uma correlação significativa entre os dois acelerómetros usados (fiabilidade inter-instrumento), um do lado direito e outro do lado esquerdo, para todos os exercícios examinados $(r=0.42$ a 0.92$)$. A diferença no dispêndio energético estimado por cada um dos aparelhos durante a marcha, subida de degraus ("stepping") e "slideboard" foi inferior a 1 $\mathrm{kcal} \cdot \mathrm{min}-1$. Foi comprovada a validade do aparelho já que houve uma correlação significativa entre o dispêndio energético estimado por cada um dos acelerómetros e a calorimetria indirecta. No entanto, a estimativa do TRITRAC foi menor do que a estimativa determinada via calorimetria indirecta, aumentando essa discrepância à medida que aumentava a carga de trabalho.

Nichols e col. (36) examinaram também a fiabilidade (inter-instrumento e inter-sessões) e a validade do TRITRAC-R3D na estimação do dispêndio energético, durante a marcha e a corrida, em 60 jovens adultos com idades entre os 18 e os 35 anos. A calorimetria indirecta foi a medida-critério de validação usada. Os coeficientes de fiabilidade dos aparelhos, usados no lado esquerdo e direito da anca, variaram entre 0.73 , a $9.7 \mathrm{~km} / \mathrm{h}$ e 0.87 , a $3.2 \mathrm{~km} / \mathrm{h}$, enquanto que os coeficientes entre as sessões demonstraram que o TRITRAC é altamente fiável de dia para dia para todas as velocidades testadas $(R=0.87-0.92)$. Relativamente à determinação da validade, os autores concluíram que os dados indicaram que o TRITRAC distingiu com precisão várias intensidades de marcha e de corrida ao nível do solo com a ressalva que não foi sensível a alterações na inclinação do tapete. No quadro 2 apresentamos o resumo dos estudos revistos sobre a validade e fiabilidade dos acelerómetros. 
Quadro 2: Resumo dos estudos revistos sobre validade e fiabilidade dos acelerómetros.

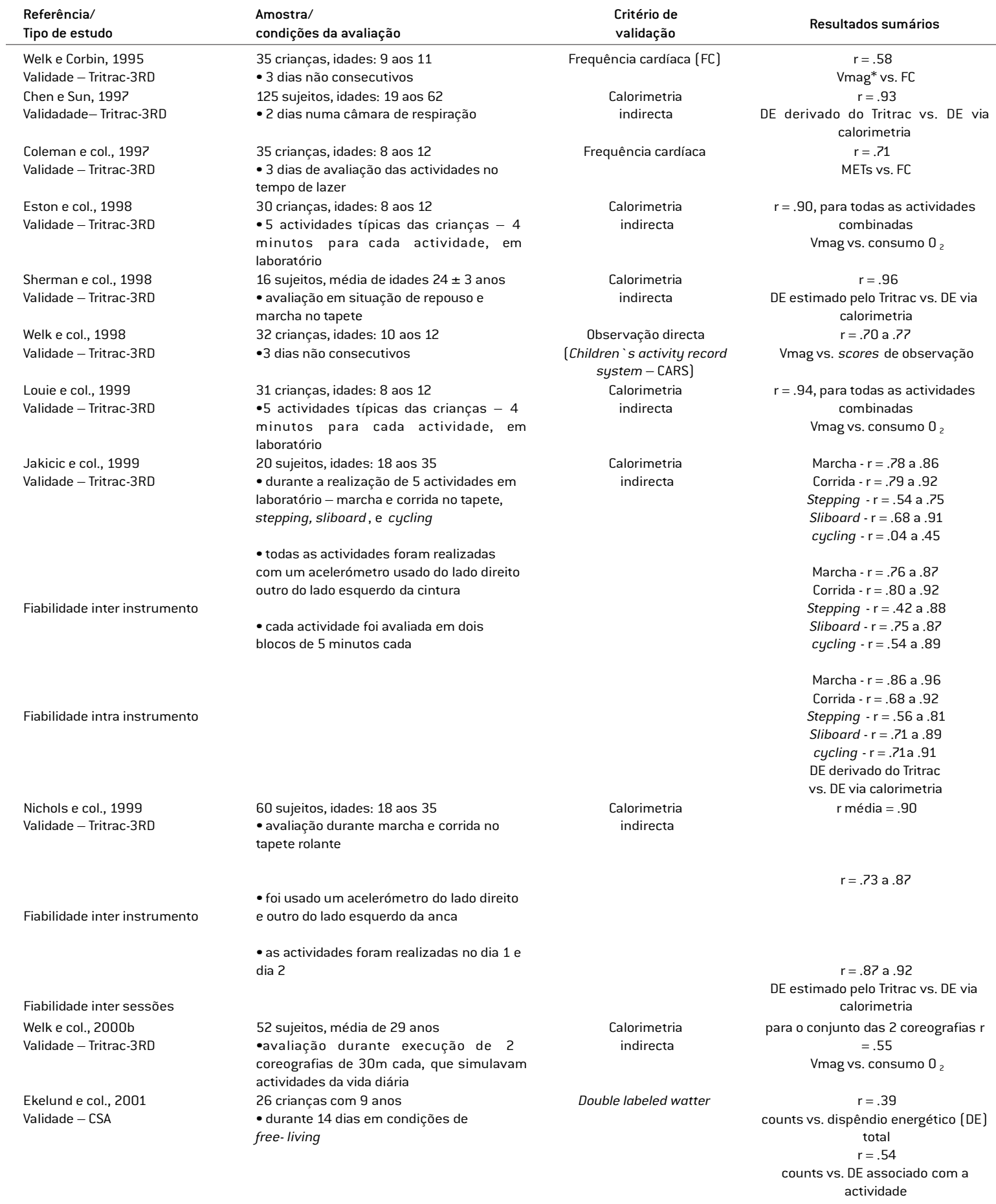

*Vmag - vector resultante; $r$ - correlação de Pearson 


\section{Pedómetros}

O pedómetro electrónico é um aparelho simples desenhado para avaliar o comportamento do indivíduo durante a marcha, efectuando o registo do número de passos dados e da distância percorrida (28). O Yamax Digi Walker - SW 700 (Yamasa Corporation, Tokyo) é um pedómetro electrónico recente que, em termos genéricos, tem um braço de alavanca horizontal que se move para cima e para baixo em resposta a oscilações verticais do corpo. Em cada passo o braço de alavanca move-se, faz um contacto eléctrico e é efectuado um registo (6). Quando se introduz o valor estimado do comprimento de uma passada e o peso do indivíduo, o aparelho fornece a distância percorrida e a estimativa do dispêndio energético para o número de passos dados (para pormenores ver 7).

A marcha é uma das formas mais comuns de actividade física e muitas vezes a que mais contribui para a actividade física diária total. É frequentemente usada para as deslocações de um lado para outro, para as actividades recreativas e para a realização das tarefas da vida diária (7). O pedómetro tem a potencialidade de providenciar, a custos reduzidos, medições objectivas e precisas da actividade física diária relativa à deslocação a pé, que por sua vez é responsável por uma fracção substancial do dispêndio calórico total na actividade física $(6,29)$. Uma das vantagens destes aparelhos é o facto de possibilitarem o registo de dados num largo período de tempo, permitindo a avaliação de padrões de actividade física num ou em vários dias. Contudo, apresentam algumas limitações quando usados como instrumentos de investigação. Embora possuam uma precisão regular na contagem dos passos, não são sensíveis a acelerações verticais acima de certos limites. Daqui que não façam distinções entre a marcha e a corrida e assumam que o sujeito gasta uma quantidade constante de energia por passo. Para o Yamax Digi-Walker o valor assumido é de cerca 0.55 $\mathrm{cal} / \mathrm{kg} /$ passo, independentemente da velocidade a que o sujeito se desloca. Outras limitações prendem-se com o facto de não providenciarem informação acerca da duração, frequência e intensidade da actividade física (5) e de não serem sensíveis à actividade que não envolva a locomoção ou que envolva o exercício isométrico (23). No entanto, os pedómetros são úteis para a distinção entre grupos com diferentes níveis de marcha, providenciando informação válida acerca deste comportamento (5).

\section{Estudos de validade e fiabilidade}

Embora pedómetros mais antigos carecessem de validade e fiabilidade, os avanços tecnológicos melhoraram a qualidade dos aparelhos e, consequentemente, da informação que providenciam. Especificamente, o modelo Yamax Digi Walker tem sido muito usado pelos investigadores, devido aos seus elevados graus de precisão e fiabilidade. Recentemente, Bassett e col. (6), num estudo de comparação da precisão de cinco pedómetros (contemporâneos) na medição da distância percorrida a pé, em adultos com idades compreendidas entre os 18 e os 65 anos, encontraram resultados aceitáveis para a maior parte dos aparelhos exibindo o Yamax Digi-Walker uma fiabilidade e validade excepcionais. Os autores mostraram que diferentes tipos de pisos não afectaram a precisão dos aparelhos e que dos cinco estudados o Yamax foi o mais preciso, tanto na medição do número de passos, como na distância percorrida, para todas as velocidades testadas. Apesar do facto de variações na velocidade de deslocação afectarem os resultados, se o indivíduo caminhar com velocidades entre os $54 \mathrm{~m} \cdot \mathrm{min}-1$ e os $107 \mathrm{~m} \cdot \mathrm{min}-1$, o pedómetro produzirá valores relativos à distância percorrida que estarão dentro de limites de erro de $20 \%$ da distância realmente percorrida. Acrescente-se que, se a velocidade de marcha for intermédia, os valores terão um erro à volta de $10 \%$ dos valores reais. Numa avaliação de terreno, ao longo de um percurso de $4.88 \mathrm{~km}$ a pé, o Yamax produziu valores de distância percorrida e passos dentro de limites de erro de $1 \%$ dos reais valores. Os investigadores referiram que alguns dos pedómetros electrónicos mais recentes mostram uma maior precisão absoluta do que os pedómetros mecânicos antigos e possuem uma qualidade elevada em estudos epidemiológicos da actividade física habitual. Referiram ainda que o pedómetro electrónico pode ser utilizado como medida-critério para validar questões sobre a distância percorrida, em questionários de actividade física.

Os resultados de Welk e col. (48), obtidos numa amostra de 31 adultos com uma média de idade de 
$29.0 \pm 8.0$, foram consistentes com os de Bassett e col. (6) em muitos registos. Ambos não encontraram diferenças no total de passos dados na marcha ou na corrida em diferentes pisos. Ambos os estudos encontraram também menor fiabilidade nas velocidades de deslocação mais baixas.

Eston e col. (20) encontraram valores de correlação entre o dispêndio energético estimado pelo pedómetro (colocado na anca) e o consumo de oxigénio medidos em crianças galesas (15 rapazes e 15 raparigas, idade média $9.3 \pm 0.8$ anos), que se situaram entre 0.78 e 0.92 para a totalidade das cinco actividades estudadas, correlação essa que foi significativamente superior às correlações correspondentes para o acelerómetro uniaxial utilizado $(r=0.85)$ e para a frequência cardíaca $(r=0.85)$. O estudo pretendeu determinar a validade da frequência cardíaca, da pedometria e da acelerometria na predição do gasto energético. Louie e col. (30) encontraram correlações muito similares embora ligeiramente superiores, num estudo com o mesmo desenho do anterior aplicado numa amostra de crianças chinesas.

Leenders e col. (29), num estudo realizado em 12 mulheres em situação de vida real, reportaram uma validade relativa do Yamax e do acelerómetro quando compararam estes dois métodos de avaliação com o PAR (Physical Activity Recall interview). O dispêndio energético relacionado com a actividade física estimado a partir do PAR foi significativamente superior ao estimado pelo TRITRAC ou pelo Yamax. Os autores chamam a atenção para o facto de existir uma grande probabilidade destas diferenças nas estimativas estarem relacionadas com o facto de cada instrumento medir diferentes formas de actividade física e de traduzirem através de processos diferentes essa informação em dispêndio energético. Referem ainda que, como não foi usada uma medida-critério "gold standard", como o caso da Doubly Labeled Water, as conclusões deverão ser entendidas com alguma cautela. Os autores salientaram que a relação entre os resultados do TRITRAC e do Yamax indicou que o número de passos registado pelo pedómetro foi representativo da actividade física diária total estimada pelo TRITRAC. Embora o pedómetro meça passos e não a intensidade do movimento corporal, a correlação entre o número médio de passos dados por dia e as contagens de actividade (vector magnitude) do TRITRAC foi significativa e elevada ( $r=0.88$ a 0.93, p<0.0001). Esta correlação evidenciou que uma grande parte da actividade física medida através do TRITRAC ficou a dever-se à deslocação a pé. Tal como atrás referimos, a quantificação da distância percorrida diariamente é um aspecto importante quando queremos avaliar a actividade física habitual, já que esta é uma das formas mais comuns de actividade e parece contribuir grandemente para o nível de actividade física diário. Recentemente, alguns investigadores reportaram que certos questionários sobre a actividade física produziam estimativas imprecisas sobre a distância diária percorrida (ver por exemplo 1, 40). Relativamente a esta questão, Bassett e col. (7) realizaram um estudo com o propósito de comparar a avaliação da distância diária percorrida entre um questionário (College Alumnus Questionnaire PAI-CAQ) e um pedómetro (Yamax). A amostra foi constituída por 96 adultos (25 aos 70 anos) de ambos os sexos com hábitos sistemáticos de actividade física. Os resultados mostraram que os indivíduos, no questionário, subestimaram a distância diária percorrida comparativamente com os resultados obtidos com o pedómetro $(1.43 \pm 1.01$ vs $4.17 \pm 1.61 \mathrm{~km} \cdot \mathrm{d}-1)$. Também os resultados relativos ao gasto de energia na marcha foram mais baixos quando obtidos com o questionário, comparativamente com os do pedómetro $(555 \pm 405 \mathrm{vs}$ $1608 \pm 640 \mathrm{Kcal} \cdot \mathrm{s}-1)$. Com o propósito de determinar a validade do aparelho, o autor realizou um estudo-piloto onde os sujeitos usaram, durante 24 horas, o pedómetro e simultaneamente, sempre que se deslocavam, empurravam uma "roda de medição". As correlações encontradas entre o pedómetro Yamax e a roda de medição foi de $r=0.977$, daqui que tenham verificado que o pedómetro providenciou uma avaliação precisa da distância percorrida. No quadro 3 apresentamos um resumo dos estudos revistos sobre a validade e fiabilidade dos pedómetros. 
Quadro 3: Resumo dos estudos revistos sobre validade e fiabilidade dos pedómetros.

\begin{tabular}{ll}
$\begin{array}{l}\text { Referência/ } \\
\text { Tipo de estudo }\end{array}$ & $\begin{array}{l}\text { Amostra/ } \\
\text { condições da avaliação }\end{array}$ \\
\hline $\begin{array}{l}\text { Basset e col., } 1996 \\
\text { Validade - Yamax DW } 500\end{array}$ & $\begin{array}{l}\text { Parte I } \\
\text { - } 20 \text { sujeitos, idades: } 18 \text { aos } 65 \\
\text { caminhar durante } 4.88 \mathrm{Km} \text { com um pedómetro } \\
\text { de cada lado da cintura }\end{array}$ \\
& \\
Fiabilidade & $\begin{array}{l}\text { Parte II } \\
10 \text { sujeitos }\end{array}$ \\
& $\frac{\bullet 400 \text { m em piso de pista e em passeio }}{\text { Parte III }}$ \\
& $\begin{array}{l}10 \text { sujeitos } \\
\text { varcha em tapete rolante a diferentes } \\
\text { velocidades }\end{array}$ \\
\end{tabular}

Eston e col., 1998

Validade - Yamax DW 200

Louie e col., 1999

Validade - Yamax DW 200

Basset e col., 2000a

Validade - Yamax DW 500

Leenders e col., 2000

Validade - Yamax DW 500

Welk e col., 2000a

Validade - Yamax DW 500
30 crianças, idades: 8 aos 12

- durante 5 actividades típicas das crianças -

4 minutos para cada actividade, com 3

pedómetros: anca, tornozelo e pulso

31 crianças, idades: 8 aos 12

- durante 5 actividades típicas das crianças -

4 minutos para cada actividade, com 3

pedómetros: anca, tornozelo e pulso

96 sujeitos, idades: 25 aos 70

- durante 7 dias excepto dormir e actividades desportivas e de lazer

17 sujeitos

- durante $24 \mathrm{~h}$

12 mulheres

- durante 7 dias em condições de free-living

31 adultos, média de idade: 20 anos

Parte I

- durante um percurso de rua e tapete rolante,

$1.61 \mathrm{Km}$, a diferentes velocidades (marcha e

corrida]

Parte II

- 7 dias para cada uma das condições:

(1) uso do pedómetro durante todo o dia excepto dormir e banho (2) uso do pedómetro durante todo o dia excepto dormir, banho e actividades físicas estruturadas

\section{Critério de} validação

Resultados sumários

Para a distância

- roda de medição ( Rolatape

Corporation, Model 400, Spokane

Para o n. ${ }^{\circ}$ de passos

- um contador manual (o sujeito carrega num manipulo em cada movimento de uma das pernas]

Para a distância

- roda de medição ( Rolatape Corporation, Model 400, Spokane WA]

\section{Calorimetria}

indirecta

Calorimetria

indirecta

College Alumnus questionnaire (PAI - CAQ)

- durante as $24 \mathrm{~h}$, sempre que se deslocavam usavam uma "roda de medição"

-7-d physical activity recall -PAR - TRITRAC - R3D

- CSA

Observação directa

7-d physical activity recall -PAR
- percentagem de passos registados pelo pedómetro $=100.7 \pm 1.5$ - $\mathrm{n} .^{\circ}$ de passos contados pelo pedómetro $=5916 \pm 568, \mathrm{n}^{\circ}{ }^{\circ}$ real de passos dados $=5874 \pm 528$ [dentro de limites de erro de $1 \%$ ]

- diferentes pisos não afectaram a precisão do pedómetro

- para velocidades entre os $54 \mathrm{~m}$. $\min ^{-1}$ e os

$107 \mathrm{~m} \cdot \mathrm{min}^{-1}$, o pedómetro produziu valores relativos à distância percorrida que estavam dentro de limites de erro de $20 \%$ da distância realmente percorrida; para

velocidade de marcha intermédia, os valores terão um erro à volta de $10 \%$ dos valores reais

CC para todas as actividades combinadas, entre consumo $\mathrm{O}_{2}$ e DE estimado pelo pedómetro Anca $=.80$

Tornozelo $=.78$ Pulso $=.67$

CC para todas as actividades combinadas, entre consumo $\mathrm{O}_{2}$ e DE estimado pelo pedómetro Anca $=.85$ Tornozelo $=.82$ Pulso $=.29$

- CC entre distância percorrida estimada pelo PAI - CAQ e pedómetro $=.41$

- CC entre distância percorrida medida pelo pedómetro e roda de medição $=.97$

CC entre DE estimado pelo PAR e DE estimado pelo pedómetro $=.94$

- CC entre counts Vmag e n. ${ }^{\circ}$ médio de passos $/ \mathrm{dia}=.93$

- CC entre counts CSA e n. ${ }^{\circ}$ médio de passos $/$ dia $=.84$

- registo do n. ${ }^{\circ}$ de passos dentro de um limite médio de erro de $10 \%$

- (1) coeficiente de correlação (CC) entre n. $^{\circ}$ médio de passos dia e dispêndio energético (DE) estimado pelo $\mathrm{PAR}=.34$

(2) CC entre n. ${ }^{\circ}$ médio de passos dia e $(D E)$ estimado pelo $P A R=.06$ 


\section{Questionários}

Os questionários representam um dos métodos mais fáceis, práticos e económicos de se estimar a actividade física, principalmente em estudos de carácter epidemiológico onde as amostras atingem grandes dimensões. Apresentam, contudo, alguns problemas de objectividade, nomeadamente devido à dificuldade dos indivíduos se recordarem com exactidão das actividades realizadas, bem como à tendência para sobrestimarem as variáveis tempo e intensidade das actividades $(28,29,35)$.

O questionário de Baecke e col. (4) é constituído por três partes distintas, cada uma reflectindo aspectos distintos da actividade física. A primeira parte pretende estimar o índice de actividade física diária no trabalho ou no tempo de escola (IAFT/E); a segunda parte é dedicada às actividades desportivas (IAFD) e a terceira visa avaliar a actividade nos tempos de lazer (IAFL). O questionário tem por objectivo estimar a actividade física total podendo esta ser determinada através do somatório dos valores encontrados em cada uma das dimensões (para pormenores ver 4).

\section{Estudos de validade e fiabilidade}

Os estudos de validade conhecidos são apresentados de forma sumária no quadro n. ${ }^{\circ} 4$. Embora nem todas as medidas critério utilizadas para estudar a validade do questionário de Baecke e col. (4) possam ser consideradas "gold standard", os resultados obtidos indiciam a enorme potencialidade que o questionário apresenta em avaliar diferentes componentes da actividade física.

Quadro 4: Resultados de estudos de validade concorrente do questionário de Baecke com outros métodos de avaliação da actividade física - adaptado de Montoye e col. (35).

\begin{tabular}{|c|c|c|c|}
\hline Outros métodos & Amostra & Coeficiente de correlação & Referência \\
\hline Acelerómetro Caltrac ( 4 dias) & 28 mulheres & $0.53^{*}$ & Mahoney \& Freedson, 1990 \\
\hline Doubly labeled water (Mets] & 28 homens & $0.32 *$ & Gretebeck e col., 1993 \\
\hline Acelerómetro Caltrac 7 dias (Mets) & & $0.52^{*}$ & \\
\hline Acelerómetro Caltrac , kcal & & $0.20 *$ & \\
\hline Large-Scale Integrated Motor & 130 mulheres & -0.11 a $0.20 *$ & Cauley e col., 1987 \\
\hline \multirow[t]{2}{*}{ Activity Monitor } & [exercitante] & & \\
\hline & $\begin{array}{l}125 \text { mulheres } \\
\text { (controlo) }\end{array}$ & 0.09 a $0.16^{*}$ & \\
\hline \multirow[t]{2}{*}{ Questionário de Paffenbarger } & $\begin{array}{l}130 \text { mulheres } \\
\text { (exercitante) }\end{array}$ & 0.09 a $0.48^{*}$ & Cauley e col., 1987 \\
\hline & $\begin{array}{l}125 \text { mulheres } \\
\text { (controlo] }\end{array}$ & 0.14 a $0.36^{*}$ & \\
\hline Ingestão calórica & 21 mulheres & $0.38^{*}$ & Albanes e col., 1990 \\
\hline Questionário de Paffenbarger & & $0.56^{*}$ & \\
\hline Questionário 7-dias & & $0.16^{*}$ & \\
\hline Questionário de Framingham & & $0.57^{*}$ & \\
\hline Questionário Minnesota Leisure time & & $0.36 *$ & \\
\hline Questionário Health Insurance Plan of New York & & $0.78 *$ & \\
\hline Questionário Lipid Research Clinics & & $0.68^{*}$ & \\
\hline Three $24 \mathrm{~h}$ recall & $\begin{array}{c}31 \text { homens e mulheres } \\
63-80 \text { anos }\end{array}$ & $0.78^{*}$ & Voorrips e col. , 1991 \\
\hline Pedómetro & & $0.72 *$ & \\
\hline Acelerómetro Caltrac , kcal ( 7 dias] & $\begin{array}{l}7 \text { homens e } 26 \\
\text { mulheres }\end{array}$ & $0.32 *$ & Miller e col., 1994 \\
\hline Questionário 7-dias & & 0.07 & \\
\hline Questionário Godin-Shephard & & $0.61 *$ & \\
\hline $\mathrm{VO}_{2}$ máx & 64 homens e mulheres & $0.54^{*}$ & Jacobs e col., 1993 \\
\hline Tapete rolante & & $0.51^{*}$ & \\
\hline Questionário Minnesota Leisure time & 73 homens e mulheres & $0.37^{*}$ & \\
\hline Acelerómetro Caltrac, Mets & & $0.19 *$ & \\
\hline Acelerómetro Caltrac, kcal & & -0.08 & \\
\hline Acelerómetro Tracmor (counts) & 134 homens com 40 & $0.47^{* *}$ & Philippaerts, 1998 \\
\hline Ingestão Calórica & anos de idade & 0.21 & \\
\hline $\mathrm{VO}_{2}$ máx & & $0.49^{* *}$ & \\
\hline Doubly labeled water & 30 homens de 40 anos & $0.69 * *$ & Philippaerts, 1998 \\
\hline
\end{tabular}


A fiabilidade do questionário foi especificamente estudada por Baecke e col. (4), Jacobs e col. (25), Pols e col. (39) e Philippaerts (38). Para além destes estudos, há que mencionar alguma pesquisa portuguesa sobre esta temática $(11,24,41)$. Todos recorreram à estimativa de fiabilidade relativa, dada pelo coeficiente de correlação intraclasse (R).

Henriques (24) testou a fiabilidade numa sub-amostra de 70 sujeitos do seu estudo, correspondendo a $13.3 \%$ da amostra total $(n=523)$ constituída por crianças e jovens dos 10 aos 17 anos. O período de tempo considerado para aplicação do reteste foi de um mês e as correlações encontradas para cada índice de actividade física em cada ano de escolaridade foram superiores a 0.80 , evidenciando a elevada fiabilidade do questionário. Os valores de $\mathrm{R}$, para todos os índices do questionário, encontrados por Sá (41) situaram-se entre 0.77 e 0.91 , o que demonstra, mais uma vez, a elevada fiabilidade do questionário. $O$ reteste foi efectuado uma semana após a $1^{\text {a }}$ aplicação em 75 sujeitos numa amostra total de 284 adolescentes do sexo masculino do $10^{\circ}$ ao $12^{\circ}$ anos de escolaridade. Cardoso (11) encontrou valores de correlação intraclasse elevados, que se situaram entre $0.78<\mathrm{R}<0.90$. O autor não referiu o intervalo de tempo nem a sub-amostra usados para o estudo da fiabilidade. A amostra total do estudo foi constituída por 786 crianças e jovens de ambos os sexos do $5^{\circ}$ ao $12^{\circ}$ anos de escolaridade, com idades compreendidas entre os 10 e os 18 anos. O quadro n. ${ }^{\circ} 5$ mostra uma síntese destes estudos.

Quadro 5: Estudos da fiabilidade do questionário da actividade física habitual de Baecke.

\begin{tabular}{|c|c|c|c|c|c|}
\hline Estudo & $\begin{array}{c}\text { Tempo entre o teste e o reteste / } \\
\text { Estatística }\end{array}$ & Amostra & & Resultados & \\
\hline \multirow[t]{3}{*}{ Baecke e col. (1982) } & 3 meses & 139 homens e & AFT & 0.88 & \\
\hline & r de Pearson & 167 mulheres & AFD & 0.81 & \\
\hline & & idades -20 a 32 & AFL & 0.74 & \\
\hline \multirow[t]{5}{*}{ Jacobs e col. (1993) } & 1 mês & 28 homens e & AFT & 0.78 & \\
\hline & rho de Spearman ajustado para a idade & 50 mulheres & AFD & 0.90 & \\
\hline & & idades -20 a 59 & AFL & 0.86 & \\
\hline & & & AFTT & 0.93 & \\
\hline & & & & 5 meses & 11 meses \\
\hline \multirow[t]{8}{*}{ Pols e col. (1996] } & 5 e 11 meses & 64 homens & $\mathrm{AFT}$ & 0.89 & 0.83 \\
\hline & r de Pearson & idades -20 a 70 & AFD & 0.88 & 0.81 \\
\hline & & & AFL & 0.76 & 0.71 \\
\hline & & & AFTT & 0.85 & 0.80 \\
\hline & & 62 mulheres & AFT & 0.80 & 0.84 \\
\hline & & idades -20 a 70 & AFD & 0.71 & 0.65 \\
\hline & & & AFL & 0.83 & 0.81 \\
\hline & & & AFTT & 0.83 & 0.77 \\
\hline \multirow[t]{4}{*}{ Philippaerts (1998] } & 1 mês & 90 homens & AFT & 0.95 & \\
\hline & coeficiente de correlação intraclasse & idades - 30 a 40 & AFD & 0.93 & \\
\hline & & & AFL & 0.87 & \\
\hline & & & AFTT & 0.86 & \\
\hline \multirow[t]{4}{*}{ Henriques (2000) } & 1 mês & 70 sujeitos & AFE & 0.81 a 0.89 & \\
\hline & coeficiente de correlação intraclasse & idades - 10 a 17 & AFD & 0.80 a 0.87 & \\
\hline & & & AFL & 0.83 a 0.94 & \\
\hline & & & AFTT & -.- & \\
\hline \multirow[t]{4}{*}{ Sá (2000) } & 1 semana & 75 sujeitos & AFE & 0.77 & \\
\hline & coeficiente de correlação intraclasse & $10^{\circ}$ ao $12^{\circ}$ anos de & AFD & 0.86 & \\
\hline & & escolaridade & AFL & 0.81 & \\
\hline & & & AFTT & 0.91 & \\
\hline \multirow[t]{4}{*}{ Cardoso (2000) } & Não referido & Não referido & AFE & 0.81 & \\
\hline & coeficiente de correlação intraclasse & & AFD & 0.87 & \\
\hline & & & AFL & 0.79 & \\
\hline & & & AFTT & 0.89 & \\
\hline
\end{tabular}




\section{EM SÍNTESE:}

- o TRITRAC é um instrumento que fornece informações acerca da intensidade, frequência e duração da actividade física. Apesar das limitações que referimos, os estudos evidenciam que é válido para avaliar a generalidade do movimento em condições de free living e o dispêndio energético associado, em populações onde a marcha e a corrida, ou outros movimentos semelhantes que desloquem o centro de massa, sejam os mais representativos do padrão de actividade e daqui permitam a comparação entre sujeitos;

- o pedómetro é um instrumento que avalia de forma objectiva a actividade física relacionada com a deslocação a pé, registando o número de passos e as estimativas da distância percorrida e da energia despendida. Os estudos de validade e fiabilidade providenciam evidência substancial acerca da validade do pedómetro, nomeadamente da marca Yamax, como um indicador objectivo da actividade física habitual;

- os questionários são os instrumentos mais práticos e comuns para a avaliação da actividade física em estudos onde as amostras adquirem proporções consideráveis, em virtude dos seus baixos custos, da sua praticabilidade e também da quantidade e detalhe de informação que é possível recolher.

Agradecimentos

Ao revisor da Revista Portuguesa de Ciências do

Desporto pelas sugestões apontadas a este artigo.

\section{CORRESPONDÊNCIA}

José António Ribeiro Maia

Faculdade de Ciências do Desporto

e de Educação Física

Laboratório de Cineantropometria

e Estatística Aplicada

Rua Dr. Plácido Costa, 91

4200 Porto

Portugal

jmaia@fcdef.up.pt 


\section{BIBLIOGRAFIA}

1. Ainsworth BE, Leon AS, Richardson MT, Jacobs DR, Paffenbarger RS (1993): Accuracy of the college alumnus physical activity questionnaire. J. Clin. Epidemiol. 46: 1403-1411. 2. Ainsworth BE, Montoye HJ, Leon AS (1994): The Consensus Statement. In: Bouchard, C.; Shephard, R., and Sthephens, T. Physical Activity, Fitness and health: International Proceedings and Consensus Statement. Human Kinetics Publishers. Champaign, Illinois.

3. Albanes D, Conway JM, Taylor PR, Moe PW, Judd J (1990): Validation and comparison of eight physical activity questionnaire. Epidemiology, 1: 65-71.

4. Baecke JAH, Burema J, Frijters JE (1982): A short questionnaire for the measurement of habitual physical activity in epidemiological studies. American Journal of Clinical Nutrition, 36: 936-942.

5. Bassett DR Jr (2000): Validity and reliability issues in objective monitoring of physical activity. Research Quarterly for Exercise and Sport, 71 (2): $30-36$.

6. Bassett DR Jr, Ainsworth BE, Leggett SR, Mathien CA, Main JA, Hunter DC, Duncan GE (1996): Accuracy of five electronic pedometers for measuring distance walked. Medicine \& Science in Sports \& Exercise, 38 (8): 1071-1077.

7. Bassett DR Jr, Cureton AL, Ainsworth BE (2000a):

Measurement of daily walking distance - questionnaire versus pedometer. Medicine \& Science in Sports \& Exercise, 32 (5): 10181023 .

8. Bouchard C, Malina R, Pérusse L (1997): Genetics of fitness and physical performance. Champaign. Human Kinetics, Illinois. 9. Bouchard C, Shephard R, Stephens T (1994): Physical activity, Fitness and health: International Proceedings and Consensus Statement. Champaign. Human Kinetics, Illinois.

10. Bouten CV, Westerterp KR, Verduin M, Janssen JD (1994): Assessment of energy expenditure for physical activity using a triaxial accelerometer. Medicine \& Science in Sports \& Exercise, 26 (12): 1516-1523.

11. Cardoso MVT (2000): Aptidão Física e Actividade Física da População Escolar do Distrito de Vila Real. Dissertação de Mestrado. Não publicada. FCDEF-UP. Porto.

12. Caspersen CJ (1989): Physical activity epidemiology: Concepts, methods and applications to exercise science. Exercise and Sport Sciences Review, 18: 423-473.

13. Caspersen CJ, Powell KE, Christensen GM (1985): Physical activity, exercise, and physical fitness: definitions and distinctions for health-related research. Public Health Reports, 100 (2): 126-131.

14. Cauley JA, LaPorte RE, Black-Sandler R, Schramm MM, Kriska AM (1987): Comparison of methods to measure physical activity in postmenopausal women. American Journal of Clinical Nutrition, 45: 14-22.

15. CDC/ACSM (1995): Physical activity and public health. A recommendation from the centers for disease control and the American College of Sports Medicine. JAMA, 273 (5): 402407.

16. Chen KY, Sun M (1997): Improving energy expenditure estimation by using a triaxial accelerometer. J. Appl. Physiolog., 83 (6): 2112-2122.

17. Coleman KJ, Saelens BE, Wiedrich-Smith MD, Finn JD, Epstein LH (1997): Relationships between Tritrac-R3D vectors, heart rate, and self - report in obese children. Medicine $\mathcal{E}$ Science in Sports \& Exercise, 29 (11): 1535-1542.
18. Crocker PRE, Holowachuk DR, Kowalski KC (2001): Feasibility of Using the Tritrac Motion Sensor Over a 7-Day Trial With Older Children. Pediatric Exercise Science, 13: 70-81. 19. Ekelund U, Sjostrom M, Yngve A, Poortvliet E, Nilsson A, Froberg K, Wedderkopp N, Westerterp K (2001): Physical activity assessed by activity monitor and doubly labeled water in children. Medicine \& Science in Sports \& Exercise, 33 (2): 275-281. 20. Eston RG, Rowlands AV, Ingledew DK (1998): Validity of heart rate, pedometry, and accelerometry for predicting the energy cost of children`s activities. J. Appl. Physiolog., 84 (1): 362-371.

21. Freedson PS, Melanson E, Sirard J (1998): Calibration of the Computer Science and Applications, Inc. accelerometer. Medicine \& Science in Sports \& Exercise, 30 (5): 777-781.

22. Freedson PS, Melanson EL (1996): Measuring physical activity. In: D. Docherty (eds). Measurement in Pediatric Exercise Science. Champaign., Illinois. Human Kinetics. pp: 261-283. 23. Freedson PS, Miller K (2000): Objective monitoring of physical activity using motion sensors and heart rate. Research Quarterly for Exercise and Sport, 71 (2): 21 - 29.

24. Henriques SCO (2000): Relação multivariada entre Actividade Física Habitual e Aptidão Física. Dissertação de Mestrado. Não publicada. FCDEF-UP. Porto.

25. Jacobs DR Jr, Ainsworth BE, Hartman TJ, Leon AS (1993): A simultaneous evaluation of ten commonly used physical activity questionnaire. Medicine \& Science in Sports \& Exercise, 25:8191.

26. Jakicic JM, Winters C, Lagally K, Ho J, Robertson R J, Wing RN (1999): The accuracy of the Tritrac-R3D accelerometer to estimate energy expenditure. Medicine \& Science in Sports \& Exercise, 31 (5): 747-754.

27. Kohl HW, Fulton JE, Caspersen CJ (2000): Assessment of Physical activity among children and adolescents: a review and synthesis. Preventive Medicine, 31(2): S54 - S76.

28. Laporte RE, Montoye HJ, Caspersen CJ (1985): Assessment of Physical Activity in Epidemiologic Research: Problems and prospects. Public Health Reports, 100 (2): 131 - 146.

29. Leenders NYM, Sherman WM, Nagaraja HN (2000):

Comparisons of four methods of estimating physical activity in adult women. Medicine \& Science in Sports \& Exercise, 32 (7): 1320-1326.

30. Louie L, Eston RG, Rowlands AV, Tong KK, Ingledew DK, Fu FH (1999): Validity of heart rate, pedometry, and accelerometry for estimating the energy cost of activity in Hong Kong Chinese boys. Pediatric Exercise Science, 11: 229-239.

31. Mahoney M, Freedson P (1990): Assessment of physical activity from Caltrac and Baecke questionnaire techniques (abstract). Medicine \& Science in Sports \& Exercise, 22: S80. 32. Maia JAR (1995): Avaliação da aptidão física. Aspectos metodológicos e analíticos. Revista Horizonte. XI 65: 190-197. 33. McArdle WD, Katch FI, Katch VL (1992): Fisiologia do Exercício: Energia, Nutrição e Desempenho Humano. Guanabara Koogan S.A., Rio de Janeiro.

34. Miller DJ, Freedson PS, Kline GM (1994): Comparison of activity levels using Caltrac accelerometer and five questionnaire. Medicine \& Science in Sports \& Exercise, 26: 376-382. 35. Montoye H, Kemper H, Saris W, Washburn R (1996): Measuring Physical Activity and Energy Expenditure. Human Kinetics. Champaign, Illinois.

36. Nichols JF, Morgan CG, Sarkin JA, Sallis JF, Calfas KJ (1999): Validity, reliability, and calibration of the Tritrac accele- 
rometer as a measure of physical activity. Medicine $\mathcal{E}$ Science in Sports \& Exercise, 31 (6): 908-912.

37. Pate RR, Long BJ, Heath G (1994): Descriptive Epidemiology of Physical Activity in Adolescents. Pedriatric Exercise Science, 6: 434- 447.

38. Philippaerts R (1998): Physical activity and physical fitness in male adolescents and adults. Dissertação de Doutoramento. Faculdade de Educação Física e Fisioterapia. Universidade Católica de Lovaina. Lovaina.

39. Pols M, Peeters P, Kemper H, Collete H (1996):

Repeatibility and relative validity of two physical activity questionnaires in elderly women. Medicine $\mathcal{E}$ Science in Sports $\mathcal{E}$ Exercise, 8 (28): 1020-1025.

40. Richardson MT, Leon AS, Jacobs DR, Ainsworth BE, Serfass $\mathrm{R}$ (1993): Comprehensive evaluation of the Minnesota Leisure Time Physical Activity Questionnaire. J. Clin. Epidemiol. 47: 271-281.

41. Sá MPCM (2000): Transmissibilidade nos Hábitos de Actividade Física. Dissertação de Mestrado. Não publicada. FCDEF-UP. Porto.

42. Sallis J, Owen N (1999): Physical Activity \& Behavioral Medicine. Sage publications, California.

43. Sherman WM, Morris DM, Kirby TE, Petosa RA, Smith BA, Frid DJ, Leenders N (1998): Evaluation of a commercial accelerometer (TRITRAC - R3D) to measure energy expenditure during ambulation. International Journal Sports Medicine, 19 : 4347.

44. Voorrips LE, Ravelli ACJ, Dongelmans PCA Deurenberg P, Van Staveren WA (1991): A physical activity questionnaire for the elderly. Medicine \& Science in Sports \& Exercise, 23: 974-979. 45. Welk GJ, Blair SN, Wood K, Jones S, Thompson RW (2000b): A comparative evaluation of three accelerometrybased physical activity monitors. Medicine $\mathcal{E}$ Science in Sports $\mathcal{E}$ Exercise, 32 (9): S489-S497.

46. Welk GJ, Corbin CB (1995): The validity of the Tritrac R3D activity monitor for the assessment of physical activity in children. Research Quarterly for Exercise and Sport, 66 (3): 202 209.

47. Welk GJ, Corbin CB, Kampert JB (1998): The validity of the Tritrac - R3D activity monitor for the assessment of physical activity: II. Temporal relationships among objective assessments. Research Quarterly for Exercise and Sport, 69 (4): 395 399.

48. Welk GJ, Differding JA, Thompson RW, Blair SN, Dziura J, Hart P (2000a): The utility of the Digi - walker step counter to assess daily physical activity patterns. Medicine $\mathcal{E}$ Science in Sports \& Exercise, 32 (9): S481-S488. 